\title{
Investigating representations of facial identity in human ventral visual cortex with transcranial magnetic stimulation
}

\author{
Sharon Gilaie-Dotan ${ }^{1,2 *}$, Juha Silvanto ${ }^{1,3}$, Dietrich S. Schwarzkopf ${ }^{1,2}$ and Geraint Rees ${ }^{1,2}$ \\ Institute of Cognitive Neuroscience, University College London, London, UK \\ 2 Wellcome Trust Centre for Neuroimaging, University College London, London, UK \\ ${ }_{3}$ Brain Research Unit, Low Temperature Laboratory, Aalto University School of Technology, Espoo, Finland
}

\section{Edited by:}

Leon Y. Deouell, The Hebrew University of Jerusalem, Israel

\section{Reviewed by:}

Gianluca Campana, Università degli

Studi di Padova, Italy;

Galia Avidan, Ben-Gurion University of

the Negev, Israel;

Joseph M. DeGutis, Harvard

University, USA

*Correspondence:

Sharon Gilaie-Dotan, Institute of

Cognitive Neuroscience and Wellcome

Trust Centre for Neuroimaging,

University College London, Alexandra

House, 17 Queen Square, London

WC1N 3AR, UK.

e-mail:s.gilaie-dotan@ucl.ac.uk
The occipital face area (OFA) is face-selective. This enhanced activation to faces could reflect either generic face and shape-related processing or high-level conceptual processing of identity. Here we examined these two possibilities using a state-dependent transcranial magnetic stimulation (TMS) paradigm. The lateral occipital (LO) cortex which is activated non-selectively by various types of objects served as a control site. We localized OFA and LO on a per-participant basis using functional MRI. We then examined whetherTMS applied to either of these regions affected the ability of participants to decide whether two successively presented and physically different face images were of the same famous person or different famous people. TMS was applied during the delay between first and second face presentations to investigate whether neuronal populations in these regions played a causal role in mediating the behavioral effects of identity repetition. Behaviorally we found a robust identity repetition effect, with shorter reaction times (RTs) when identity was repeated, regardless of the fact that the pictures were physically different. Surprisingly, TMS applied over LO (but not OFA) modulated overall RTs, compared to the No-TMS condition. But critically, we found no effects of TMS to either area that were modulated by identity repetition. Thus, we found no evidence to suggest that OFA or LO contain neuronal representations selective for the identity of famous faces which play a causal role in identity processing. Instead, these brain regions may be involved in the processing of more generic features of their preferred stimulus categories.

Keywords: OFA, LO, faces, famous, familiar, identity, state-dependent TMS

\section{INTRODUCTION}

Humans can recognize the identity of a familiar face in many different contexts and from many different viewpoints. This involves overcoming abundant visual variations in an individual's appearance such as viewpoint, hairstyle, and facial expression. How this is achieved in the human brain remains unclear. The neural substrates of face processing involve a complex network of facesensitive regions [e.g., the fusiform face area (FFA, Kanwisher et al., 1997; McCarthy et al., 1997; Halgren et al., 1999; Levy et al., 2001), the occipital face area (OFA, Gauthier et al., 2000b; Hasson et al., 2003; Gilaie-Dotan and Malach, 2007), a region in the superior temporal sulcus (STS, Haxby et al., 2000; Andrews and Ewbank, 2004; Winston et al., 2004; Pourtois et al., 2005), and a region in the anterior temporal pole (Kriegeskorte et al., 2007)]. But it is still unclear whether these brain regions are involved in determining facial identity. At anatomically early stages of this network, the OFA shows preferential activation to faces over other object categories (Halgren et al., 1999; Haxby et al., 1999; Gauthier et al., 2000b; Gilaie-Dotan et al., 2008). Although some studies have implied that the involvement of OFA in face representation is limited to processing of facial features (Liu et al., 2009), or their spatial configuration (Rotshtein et al., 2007; Rhodes et al., 2009), others have suggested the involvement of OFA in higher levels of facial processing (Chen et al., 2007) including its necessity for facial recognition, as patients with a lesion overlap in the right OFA exhibit face recognition deficits (Rossion et al., 2003; Steeves et al., 2006, 2009). Thus, the face-related activation in the OFA region may arise from the processing of low-level face or shape features that face images contain (Lerner et al., 2008; Dakin and Watt, 2009; Liu et al., 2009), or alternatively, this region may contain neuronal representations that encode face identity (independently of low-level features).

Here we sought to investigate these issues by using state-dependent transcranial magnetic stimulation (TMS, Silvanto and Muggleton, 2008a) to investigate whether OFA contains representations of facial identity (independently of low-level visual attributes) that play a causal role in identity detection. State-dependent TMS makes use of the phenomenon that TMS can either facilitate or disrupt cognitive functions depending on the initial state of the stimulated neuronal population. By bringing functionally distinct neuronal populations within a cortical region to different initial states (using manipulations such as adaptation, repetition, or priming), TMS can differentially affect these distinct populations despite of their spatial overlap (see Silvanto and Muggleton, 2008a,b; Silvanto et al., 2008 for reviews). State-dependent TMS can be used to investigate neural tuning in the targeted region. Specifically, if TMS differentially affects the processing of an attribute depending on whether that attribute has been repeated/adapted, this indicates that the targeted region contains neuronal representations that were affected by (and thus tuned to) the attribute. By contrast, if no differential effects are found, this indicates that the initial state manipulation did not 
modulate neural activity in that region, suggesting a lack of neural tuning to the manipulated attribute. The benefit of this paradigm is that it combines spatial specificity and functional resolution with the ability to assess causality. This paradigm has already been successfully used to investigate neuronal representations of highlevel attributes such as numbers (Cohen Kadosh et al., 2010) and semantic categories (Cattaneo et al., 2010). Furthermore, we have recently demonstrated that this method can successfully distinguish neuronal populations in the regions investigated in this study [OFA and lateral occipital (LO)] with respect to rotational invariance of two-dimensional shape detection (Silvanto et al., in press).

To examine whether OFA contained populations of neurons tuned for facial identity and causally involved in identity processing, we used an identity repetition paradigm to modulate the initial state of neurons that are selective for face identity. We examined participants while they attempted to determine whether two successively presented but physically different face images were of the same or different individuals. In such situations, people respond faster when the same identity is repeated compared to when a different identity is presented, thus demonstrating facial identity repetition facilitation (Johnston and Barry, 2001). Importantly, this facilitation is achieved even when the images of the same identity are physically dissimilar. This demonstrates the presence of neuronal populations in the brain which are sensitive to facial identity and that their activation state is influenced by previously viewed identity. Viewing a particular face presumably elicits activity in corresponding identity-specific neuronal representations, thus facilitating the processing of consequent views of the same individual.

To ensure that these repetition of identity effects depended on facial identity alone and not on low-level image similarities, we deliberately sought to remove any effect of repetition of low-level image properties by choosing to examine repetition of physically very different face images from the same individual. We reasoned that if identity-based neuronal representations existed in OFA, identity exposure would influence the initial activation state of these representations. TMS was then applied over OFA during the delay period between the first face image and the subsequent target face image, which required a behavioral response (e.g., Cattaneo et al., 2010). If identity-based neuronal representations were present in this area, we hypothesized that they would be activated by the first face image, and so TMS application after the first face image would facilitate neural populations encoding this identity representation, as TMS has been shown to facilitate neuronal representations held in working memory (Cattaneo et al., 2009). If such populations played a causal role in any subsequent behavioral benefit ("identity repetition effect") then we would see an impact of TMS on the identity repetition effect. We therefore compared the effect of TMS on responses elicited by the second face image when that representation was activated by the first image (i.e., "same identity" trial) relative to trials on which the first and second image activated distinct identity representations. In contrast, if there were no identity-specific representations in OFA, a differential TMS effect on the same/different trials would not be seen. The experimental logic was thus similar to fMR-adaptation in which the BOLD induced by a second stimulus can be modulated by the presentation of the first stimulus if the two are encoded by the same neuronal population.
The LO region (Malach et al., 1995; Grill-Spector et al., 1998a; Lerner et al., 2001) served as a control site since it is non-selectively activated by objects and faces (Avidan et al., 2002b; Fang et al., 2007; Gilaie-Dotan et al., 2008, 2010; Lerner et al., 2008). Furthermore it is in the vicinity of OFA yet functionally separable from OFA as has been previously shown in TMS studies (Pitcher et al., 2009).

\section{MATERIALS AND METHODS PARTICIPANTS}

Twelve healthy participants [six males and six females, aged $26.8 \pm 6.6(\mathrm{SD})]$ took part in the experiment. All had normal or corrected-to-normal vision. All participants gave written informed consent before participating in the study, which was approved by the local ethics committee (Institute of Neurology and National Hospital Joint Ethics Committee), and were treated in accordance with the Declaration of Helsinki.

\section{STIMULI}

All images were identified through Google Image search based on identity query. Images were processed in Adobe Photoshop 6.0 to be of identical dimensions including: cropping original image to a height:width ratio of 5:4 and then resizing to 300 pixels $($ width $) \times 375$ pixels (height). Text was removed. Images were categorized into different groups according to gender, then skin color, and then hair color (see Table 1 for specific groups). Pairs of images for different identity trials were constructed so that both were taken from the same group to minimize basic level cues to guide or bias behavior (i.e., that same-different decisions difficulty will not be based on basic level cues).

\section{PIXEL-WISE DIFFERENCES ANALYSIS}

For each pair of images $(K, J)$, for each color channel $(\mathrm{R}, \mathrm{G}, \mathrm{B})$ separately, we calculated the pixel-wise inter-pair channel difference (Euclidean distance) as $d(K, J)=(1 / \sqrt{\text { imsize }}) \sqrt{\Sigma(K-J)^{2}} s$ with $K, J$ measured in $(0,255)$ units. The average over the three channels was then taken as the inter-pair difference. For grayscale images (singlechannel image) the image itself was considered for each channel comparison. For each experimental block averages were calculated separately for the repeated identity pairs and for the different identity pairs in the block. Lastly these averages over all six blocks were taken as the mean pixel-wise difference for repeated identity and different identity pairs (see Figures 2B,D). To determine significant differences between the pair types two-tailed $t$-tests (paired, equal variance and unequal variance) were applied to these pixel-wise differences averages (repeated vs. different pairs) of all the blocks Additionally, two-tailed $t$-tests were also applied to the raw pixelwise differences of repeated and different pairs (150 repeated pairs vs. 150 different pairs).

Normalization of the images (see Figure 2C) was performed by first defining a circular region encompassing the face only for each of the images, while the rest of the image was set to white. The image was then cropped to the minimal square containing the face image circle. Images were then rescaled to a size of $150 \times 150$ pixels (to enable comparable face calculations). Pixel-wise analysis (Figure 2D) was calculated on these images in the same manner as described above. 
Table 1 | Individuals used as stimuli grouped by gender, complexion, and hair color.

\begin{tabular}{|c|c|c|c|c|c|c|}
\hline \multicolumn{2}{|c|}{ Males } & \multicolumn{5}{|c|}{ Females } \\
\hline \multicolumn{2}{|c|}{ Light skin } & Dark skin & \multicolumn{3}{|c|}{ Light skin } & Dark skin \\
\hline Bill Clinton & Al Pacino & Barack Obama & Angela Merkel & Annette Bening & Andie MacDowell & Beyonce \\
\hline Bill Gates & Antonio Banderas & Denzel Washington & Britney Spears & Celine Dion & Angelica Houston & Michelle Obama \\
\hline David Beckham & Bruce Willis & Michael Jordan & Hillary Clinton & Shirley MacLaine & Cherie Blair & Tina Turner \\
\hline David Bowie & George Bush & Tiger Woods & Jessica Parker & Susan Sarandon & Courtney Cox & \\
\hline Donald Trump & David Schwimmer & Nelson Mandela & Kim Basinger & Emma Watson & Cynthia Nixon & \\
\hline Elton John & Robert De Niro & Eddie Murphy & Kim Cattrall & & Demi Moore & \\
\hline Vladimir Putin & Dustin Hoffman & & Laura Dern & & Geena Davis & \\
\hline Albert Einstein & Gerard Depardieu & & Lisa Kudrow & & Penelope Cruz & \\
\hline \multirow{12}{*}{ Brad Pitt } & Jeff Goldblum & & Meg Ryan & & & \\
\hline & Jerry Seinfeld & & Melanie Griffith & & & \\
\hline & John Lennon & & Meryl Streep & & & \\
\hline & John Travolta & & Olivia Newton-John & & & \\
\hline & Keanu Reeves & & Scarlett Johansson & & & \\
\hline & Cosmo Kramer & & & & & \\
\hline & (Michael Richards) & & & & & \\
\hline & Matt Leblanc & & & & & \\
\hline & Matthew Perry & & & & & \\
\hline & Nicolas Cage & & & & & \\
\hline & Prince Charles & & & & & \\
\hline & Van Damme & & & & & \\
\hline
\end{tabular}

\section{EXPERIMENTAL SETUP}

All stimuli were presented centrally on an SVGA 17 inch monitor set at $1024 \times 768$ resolution and a refresh rate of $60 \mathrm{~Hz}$. Stimuli were viewed from $41 \mathrm{~cm}$ distance subtending a visual angle of $9.35^{\circ} \times 12.38^{\circ}$ (width $\times$ height .

On each trial, the first face image was presented for $1000 \mathrm{~ms}$, and after a delay of $500 \mathrm{~ms}$ the second face image (target stimulus) appeared for $200 \mathrm{~ms}$. In TMS trials the TMS pulse train was applied during the delay period (see details above). The first face stimulus and the second face stimulus were always different images. On "repeated identity" trials, the second face image was the same individual as in the first face. On "different identity" trials, the second face was a different individual than the first face. Participants were instructed to respond as fast as they could in a same - different forced choice task whether the individual depicted by second face was the same (respond "same") or different than (respond "different") the individual depicted by the first face.
The presentation of the second target stimulus was chosen to be short $(200 \mathrm{~ms})$ in order to eliminate possibility of eye movements across the target and to avoid ceiling effect in performance levels. The participant's response was expected after the target stimulus went off. Note however that even immediate responses following the target's onset were highly likely to actually occur after the target's offset (200 ms) since reaction times (RTs) are usually longer than $350 \mathrm{~ms}$ and thus those responses would have been recorded.

The experiment was run in six blocks of 50 trials each [two blocks for each of the TMS conditions (OFA-TMS, LO-TMS, or No-TMS)], the order was randomized and counter balanced across participants. In each block, there were 25 repeated identity and 25 different identity trials. There were thus a total of 50 repeated and 50 different identity trials for each TMS condition. 
The 25 repeated identity trials in each block were made of 25 different identities. Furthermore, identities were usually not repeated within a block (repeated or different), such that each block generally consisted of 75 different identities, except for six cases. In three blocks there were 75 different identities, in two blocks 73 different identities, and in one block 72 different identities. Overall in the experiment 85 identities were used, each appearing $5.29 \pm 0.13$ times throughout the experiment. A total of 359 different images were used in the experiment, out of which 90 of them were used more than once (in most cases the image was used once in a repeated identity trial in a certain block) and again in another different identity trial in a different block.

\section{fMRI LOCALIZATION OF OFA AND LO}

A 1.5T Siemens Sonata system was used to acquire T1-weighted anatomical images and $\mathrm{T} 2{ }^{\star}$-weighted echo planar images (EPIs) with BOLD contrast. Each EPI comprised of thirty-two 2-mm axial slices with a 1-mm inter-slice gap covering the whole cerebrum with an in-plane resolution of $3 \mathrm{~mm} \times 3 \mathrm{~mm}$. The experiment was split into five runs, each consisting of 78 volumes. The first four volumes of each run were discarded to allow for T1 equilibration effects. Volumes were acquired continuously with a TR of $2.88 \mathrm{~s}$ per volume.

The functional localizer scan used a one-back paradigm to focus attention on the stimuli. The three categories of visual stimuli: faces, objects, and scrambled images of the objects. Each image was presented for $360 \mathrm{~ms}$ with a 360-ms blank interval between images. Participants were instructed to press a key whenever they detected two images repeated in a row (one-back task) to ensure they were alert and attentive. Stimuli were presented in blocks of 40 items from within a category, and a centrally presented red fixation cross was present throughout the experiment. The serial position of the categories was varied and all blocks were repeated, using a total of 80 different images per category.

All images were grayscale. In order to minimize retinotopic effects, we first generated phase-scrambled versions of the original images of all objects and faces and then superimposed each image onto these scrambled images. Object stimuli created in this manner were further phase-scrambled to generate the scrambled category. This ensured that each category occupied the same area of visual space and that the spatial frequency and orientation content of objects and scrambled objects was identical. Functional imaging data were analyzed using SPM5 (http:// www.fil.ion.ucl.ac.uk/spm). After deleting the first four volumes of each run to allow for T1 equilibrium effects, the functional images were corrected for slice acquisition time, realigned to the first image using an affine transformation to correct for small head movements and EPI distortions unwarped using B0 field maps. The images were then smoothed with an 8 - $\mathrm{mm}$ full-width half-maximum Gaussian filter and pre-whitened to remove temporal autocorrelation. The resulting images were entered into a participant-specific general linear model with three conditions of interest corresponding to the three categories of visual stimuli. For each run blocks were convolved with a canonical hemodynamic response function to generate regressors. In addition, the estimated motion parameters were entered as covariates of no interest, to reduce structured noise due to residual head motion effects. Linear contrasts among the condition-specific regressors taking into account all five runs were used to identify the two TMS target sites within each participant's right hemisphere: OFA by contrasting activation associated with face presentation to object presentation, and LO by contrasting objects to scrambled objects. The functional images were then registered to each participant's individual structural scan using a 12-parameter affine transformation to identify two TMS target sites (rOFA, rLO) in the right hemisphere.

Each TMS target site was individually identified in each participant by selecting the peak activation voxel for each contrast (see above) in the LO aspect of the cortex (see Figure $\mathbf{1}$ for average beta coefficients of peak voxels). The target sites corresponded well with previously reported maps of object- and face-selective regions (Hasson et al., 2003; Pitcher et al., 2009). Coordinates of the target sites in Talairach space for all participants are listed in Table 2.

\section{TRANSCRANIAL MAGNETIC STIMULATION}

A Magstim Rapid stimulator (Magstim, Wales) and a 70-mm figure-of-eight coil was used for stimulation. A fixed TMS intensity $(60 \%)$ was used on the basis of a number of previous studies (e.g., Campana et al., 2002; Silvanto et al., 2005; Pitcher et al., 2007). In the TMS conditions, a $10-\mathrm{Hz}$ pulse train consisting of three pulses was applied on each trial during the delay between the first face stimulus and the second face stimulus. The first pulse of the pulse train was applied $300 \mathrm{~ms}$ after the offset of the first face stimulus, so that the pulse train ended with the onset of the second face stimulus. The coil orientation was such that the coil handle was pointing upwards and parallel to the midline.

Stimulation sites were localized using the Brainsight TMS-MRI co-registration software (Rogue Research), utilizing individual high-resolution MRI scans for each participant. The right OFA and right LO were localized by overlaying individual activation maps from the fMRI face and object localizer experiment, and the coil locations were marked on each participant's head. The target area was identified by selecting the voxel exhibiting the peak activation in each functionally defined area (see details of functional localizers below).

\section{RESULTS}

\section{CONTROLLING FOR LOW-LEVEL IMAGE SIMILARITIES}

Since familiar and unfamiliar faces are processed differently in the human brain (Hancock et al., 2000), we chose a uniformly familiar stimulus set comprising only of well known individuals so that all participants would be familiar with their identity (see Table 1 for a complete list of the celebrities used and their groupings). Since low-level image similarities could potentially influence any behavioral facilitation effects, as images of the same individual might be much more similar in their low-level properties than images of different individuals, we deliberately minimized such effects. First, we grouped individuals according to the same gender, skin complexion and hair color. Figure $2 \mathrm{~A}$ displays examples of stimuli of primed-identity and non-primed identity trials. We then calculated pixel-wise image differences for each of the conditions ("repeated identity" and "different identity") to verify that images of the "repeated identity" and the "different identity" conditions were comparable with respect 


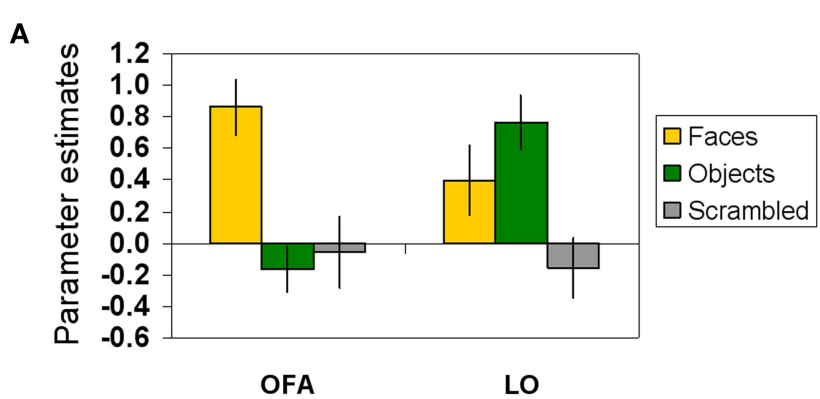

B
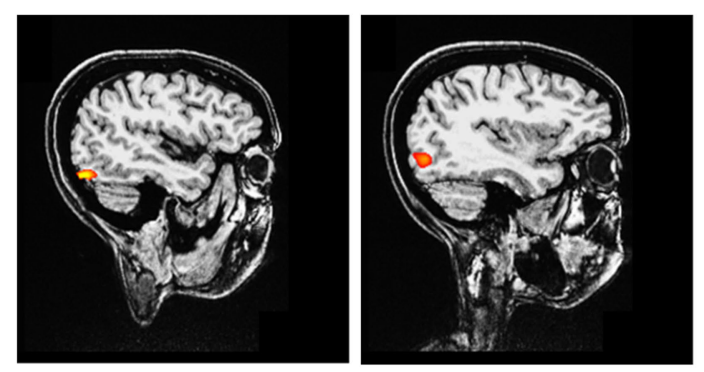

OFA-R

LO-R

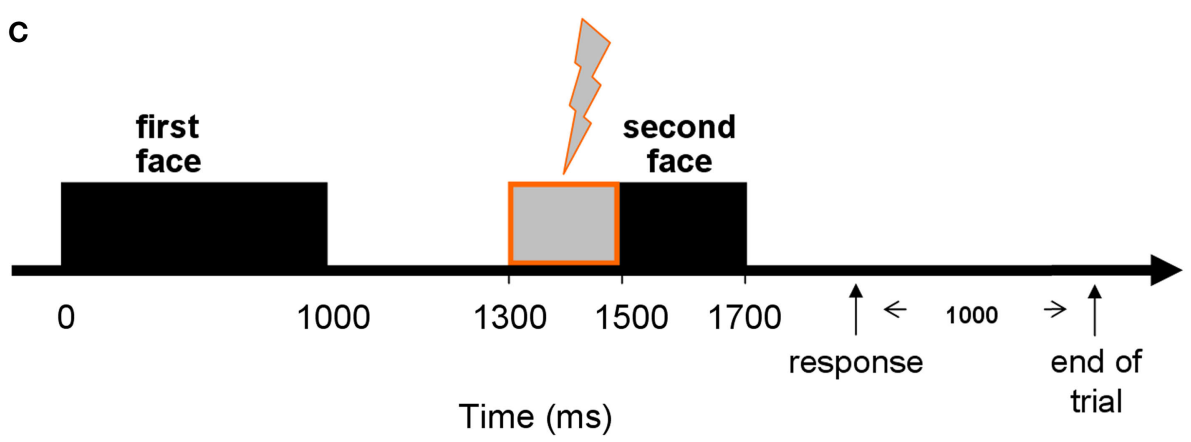

FIGURE 1 | Localization of the TMS sites by fMRI localizer and TMS trial. (A) fMRI localizer average model parameter estimates of OFA-R's and LO-R's peak voxels that were used to define per-participant coordinates for the TMS sites (OFA: faces-objects, LO: objects-scrambled). (B) OFA-right and LO-right foci of a representative participant. (C) Experimental TMS paradigm: time line of a single trial. The first face image was presented for $1000 \mathrm{~ms}$, followed by a delay of $500 \mathrm{~ms}$ after which the target image was presented for $200 \mathrm{~ms}$ (see Materials and Methods for details). ATMS train of three pulses was applied during the delay, starting at $300 \mathrm{~ms}$ after the first image offset and ending with the target onset. TMS was applied to OFA, to LO, or not at all. Thousand milliseconds after the participant responded (pressing a button to indicate whether the two images represented the same person or a different person), the trial ended and a new one began. to their low-level image statistics. These results are displayed in Figure 2B (see Materials and Methods for further details). As we hoped, pixel-wise differences were very similar and statistically indistinguishable for both conditions $[t(10)=0.133, p>0.87$, two-tailed $t$-test over block averages, and $t$-tests over all pairs of images (repeated vs. different), $t(298)=0.16, p>0.87$ ]. However, since the images we used included a wide variety of backgrounds that could conceivably contribute to any differences we observed, we further sought to examine whether low-level image differences between the two conditions might be revealed when only the part of the image corresponding to the individual face was analyzed. We therefore limited a second analysis to only the facial part of each image (see Figure 2C, and additional details in Materials and Methods) and, after normalizing the faces to a common size, calculated the pixel-wise differences taking only these facial regions into account (Figure 2D). This further confirmed that there were no significant low-level differences between the conditions [repeated identity vs. different identity, $t(10)=0.405, p>0.69$, two-tailed $t$-test over block averages, and $t$-tests over all pairs of normalized images (repeated vs. different), $t(298)=0.452, p>0.65]$, even when the analysis was confined to the normalized face regions only.

\section{REACTION TIME ANALYSIS}

Participants performed an identity-matching task. They were presented with an image of a face, and after a short delay saw a different face image (probe). They had to respond as fast as possible with a button press to indicate whether or not the probe showed the same individual as the first image. A short TMS train was applied during the delay period between the first image and the probe ending at the onset of the probe. TMS was applied either to OFA, LO, or not at all (see Figure 1) in different blocks. 
Table 2 | Talairach (MNI) coordinates of TMS target sites in each participant.

\begin{tabular}{|c|c|c|c|c|c|c|}
\hline Participant & \multicolumn{3}{|c|}{ LO } & \multicolumn{3}{|c|}{ OFA } \\
\hline 1 & $55(63)$ & $-70(-69)$ & $20(27)$ & $50(58)$ & $-82(-81)$ & $-1(4)$ \\
\hline 3 & $44(51)$ & $-87(-86)$ & $5(11)$ & $45(52)$ & $-78(-77)$ & $-7(-3)$ \\
\hline 4 & $35(41)$ & $-95(-95)$ & $-13(-9)$ & $35(41)$ & $-80(-79)$ & $-13(-10)$ \\
\hline 5 & $46(53)$ & $-72(-71)$ & $-17(-15)$ & $18(21)$ & $-98(-98)$ & $-11(-6)$ \\
\hline 7 & $41(48)$ & $-78(-77)$ & $6(12)$ & $39(45)$ & $-93(-93)$ & $0(5)$ \\
\hline 8 & $46(53)$ & $-77(-76)$ & $-3(1)$ & 49 (57) & $-65(-64)$ & $-12(-9)$ \\
\hline 9 & $34(40)$ & $-82(-81)$ & $2(8)$ & $45(53)$ & $-79(-78)$ & $-13(-10)$ \\
\hline 10 & $35(41)$ & $-83(-82)$ & $-8(-4)$ & $23(27)$ & $-92(-92)$ & $-18(-14)$ \\
\hline 11 & $40(47)$ & $-71(-70)$ & $-11(-8)$ & $53(61)$ & $-64(-63)$ & $5(10)$ \\
\hline
\end{tabular}

Note that the average of the intra-subject distance between LO and OFA location in Talairach space was $19.2 \pm 7 \mathrm{~mm}$ (21 $\pm 8 \mathrm{~mm}$ in MNI space). Error bars, SD.

A robust identity repetition modulation effect was found with faster RTs in the repeated identity trials $[676 \pm 34 \mathrm{~ms}(\mathrm{SEM})]$ compared to the different identity trials $[730 \pm 46 \mathrm{~ms}(\mathrm{SEM})]$ in the No-TMS condition and this difference was statistically significant $[t(11)=3.204, p=0.0083$, correct trials $]$. This significant reduction in RT was evident in all 12 participants and can be seen in Figure 3A (No-TMS condition).

This identity repetition modulation effect remained strong and significant even when we applied TMS to LO or OFA (Figure 3A). A repeated-measures ANOVA with identity repetition (repeated, different) and TMS site (No-TMS, LO-TMS, OFA-TMS) on RTs of correct trials revealed a significant main effect of identity repetition $[F(2,22)=12.347, p=0.0049]$ evident in the shorter RTs for repeated identity trials. For OFA-TMS this identity repetition effect was evident in 9 out of the 12 participants [difference between primed and non-primed trials $35 \pm 13 \mathrm{~ms}$ (SEM)], and for LO-TMS in 10 of the 12 participants [difference between repeated and different trials of $43 \pm 14 \mathrm{~ms}$ (SEM)]. Figure 3B displays the raw RT values for each of the conditions.

TMS had a site-specific effect on overall RTs. This was supported by a significant effect of TMS site $[F(2,22)=3.472, p=0.0489]$. Post hoc analysis revealed that surprisingly, RTs in the LO-TMS condition, but not in the OFA-TMS condition, were significantly different from those in the No-TMS condition, as shown by Figure 3B (Bonferroni/Dunn: $p=0.0152$; for 10 of the 12 participants there was a reduction in RT in repeated identity LO-TMS trials vs. No-TMS trials; in 8 of the 12 participants for different trials). This reduction in RTs was not a general TMS effect since RTs for OFA-TMS were not significantly different than for the No-TMS condition (Bonferroni/Dunn: $p=0.1714$; only half of the participants showed an average reduction in RT when TMS over OFA was applied for repeated and different trials).

TMS modulation of the identity repetition effect would indicate that the underlying population was sensitive to identity. However, the interaction between TMS site and identity repetition was not significant $[F(2,22)=1.003, p=0.383]$. Further post hoc tests did not reveal any differences between the modulation of TMS on repeated trials vs. different trials for either LO or OFA [two-tailed paired $t$-test: LO-TMS vs. No-TMS, repeated: $t(11)=2.819, p=0.017$, different: $t(11)=2.231, p=0.047$; OFATMS vs. No-TMS, repeated: $t(11)=0.756, p=0.466$, different: $t(11)=1.303, p=0.219$ ].

Thus, the reduction in RTs induced by TMS over LO was independent of identity repetition. This indicates that neuronal populations in LO affected by TMS were related to general face or shape processing but were not specifically related to facial identity representations.

\section{ACCURACY ANALYSIS}

The accuracy levels of the repeated identity trials [ $92.8 \pm 1.7 \%$ $(\mathrm{SEM})]$ and the different identity trials $[89.5 \pm 3 \%(\mathrm{SEM})]$ were high and no significant differences between trial types were found [No-TMS: $t(11)=1.24, p=0.2401$; five participants were more accurate in the repeated identity trials, five participants were more accurate in the non-repeated trials, and two participants showed identical performance in the two trial types; see Figure 4A]. Accuracy levels remained high when TMS was applied to OFA (repeated identity: $89.8 \pm 2.1 \%$, different identity: $89.3 \pm 2.5 \%$ ) or to LO (repeated identity: $90 \pm 2.4 \%$, different identity: $89.7 \pm 2.7 \%$ ). To analyze the effects of TMS on accuracy we carried out a repeated measured ANOVA on accuracy levels. No main effects or interaction were significant [identity repetition: $F(2,22)=0.459$, $p=0.511$; TMS site: $F(2,22)=2.383, p=0.1157$; interaction $F(2,22)=0.834, p=0.448]$. However planned pair-wise dedicated comparisons revealed a significant LO-TMS effect for repeated identity trials only relative to the No-TMS condition [two-tailed paired $t$-test: repeated: $t(11)=2.225, p=0.048$ ] As can be seen in Figures 4A,B, this was evident by the reduction in accuracy for repeated trials in LO-TMS relative to the No-TMS condition [average reduction of $2.8 \pm 1.26 \%$ (SEM), which was observed in 8 out of the 12 participants]. 


\section{$\mathbf{A}$} repeated identity first face second face
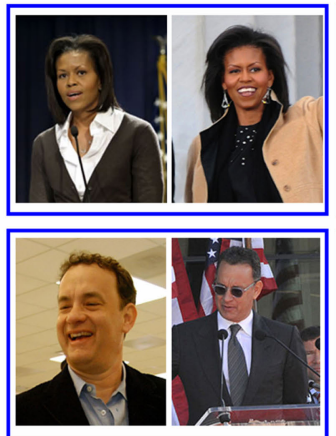

B

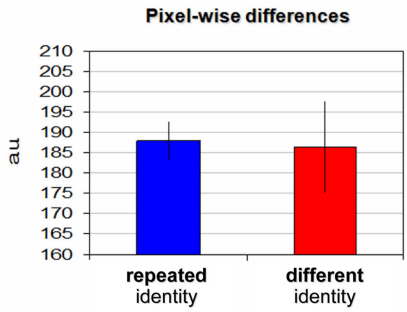

C

repeated identity first face second face
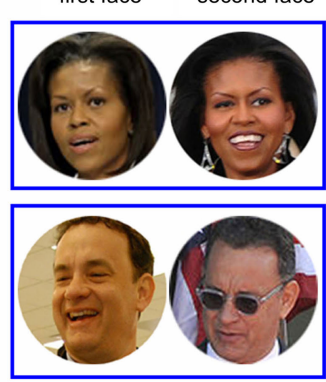

D

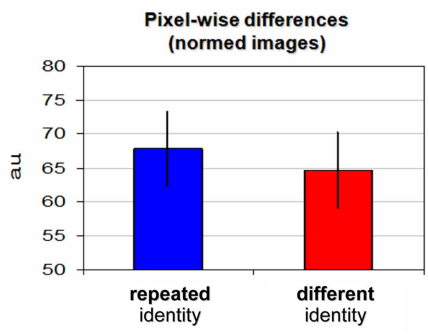

FIGURE 2 | Stimuli examples and pixel-wise differences. (A) Examples of repeated identity trials stimuli (blue frame) and different identity trials stimuli (red frame). The first presented face image appears on the left, the second on the right. Repeated identity trials depicting Michelle Obama (top) and Tom Hanks (bottom). Different identity trials depicting Al Pacino, Dustin Hoffman, Britney Spears, and Scarlett Johansson are shown. See the list of individuals in Table 1; please note that these are examples for illustrative purposes and the exact images used in the experiment are available on request from the authors. (B) Pixel-wise differences for repeated identity trials and different identity trials (see further details in Materials and Methods). No significant difference in low-level image differences was found between the trial types. (C) Examples from the post hoc stimuli analysis: all stimuli were cropped to restrict the picture to the face only and normalized to a common size (see further details in Materials and Methods). (D) Pixel-wise differences as in (B) for the normalized images (described in C).

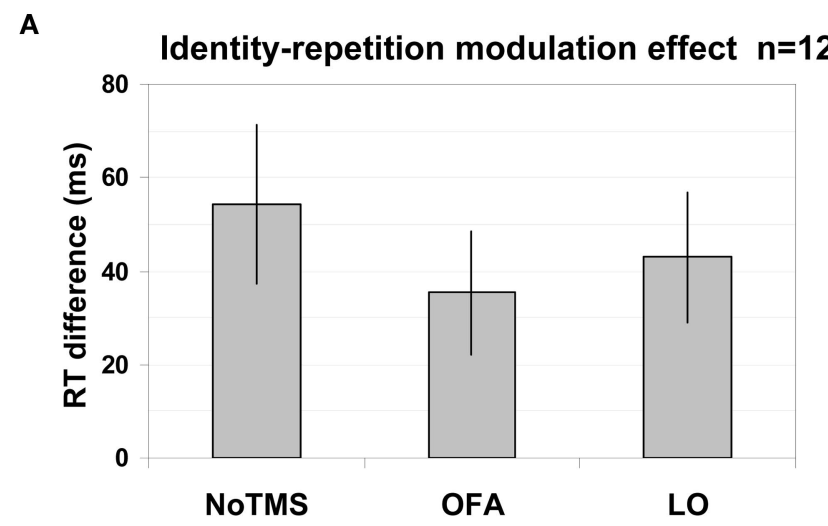

B

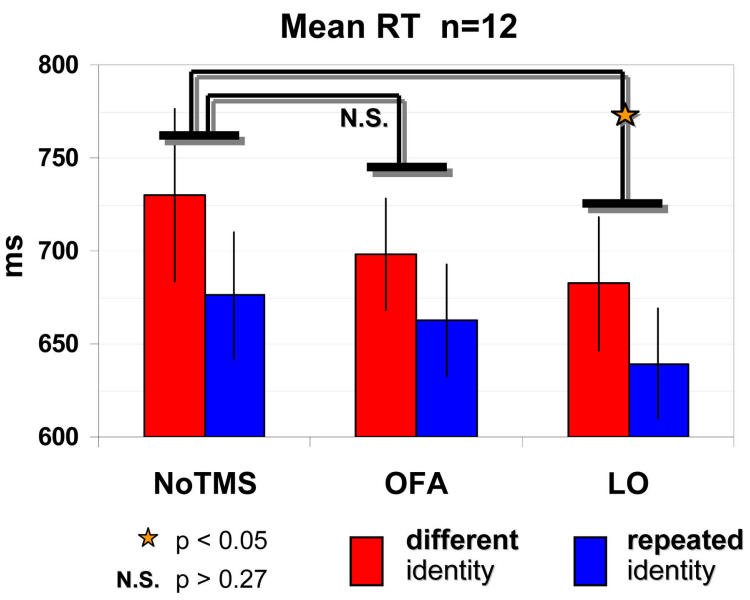

FIGURE 3 | Identity repetition modulation effect and LO site-specific effect as evident by shorter RTs. (A) Significant identity repetition modulation effect: Reduction in RTs (correct trials) when identity is repeated compared to different trials, regardless of the TMS site. (B) Mean RT for correct trials according to trial type andTMS site. TMS site effect for LO: LO-TMS was significantly different than No-TMS while OFA was not, and this was true for both different identity trials and repeated identity trials. Red - different identity trials, blue - repeated identity trials. Error bars, SEM.

For non-repeated trials in LO-TMS no significant change was observed relative to No-TMS condition [two-tailed paired $t$-test: $t(11)=0.129, p=0.9$; average change of $-0.17 \pm 1.29 \%$ (SEM)]. For OFA-TMS neither conditions showed significant different relative to No-TMS condition [repeated trials: $t(11)=1.6, p=0.138$, average reduction $2.97 \pm 1.86 \%$ (SEM), observed in 6 of the 12 participants; non-repeated trials: $t(11)=0.167, p=0.87$, average reduction $0.17 \pm 0.99 \%$ (SEM) in 4 of the 12 participants].

\section{DISCUSSION}

In this study we used state-dependent TMS to investigate whether face-related activity in OFA might reflect the presence of neuronal populations sensitive to facial identity in this region, while LO served as a control site. We used a stimulus set in which low-level 


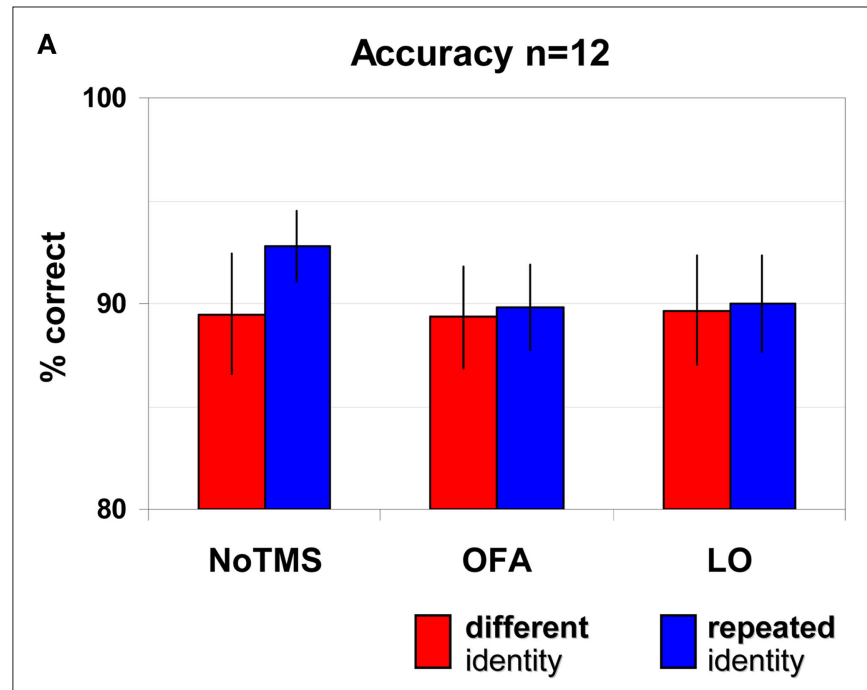

B
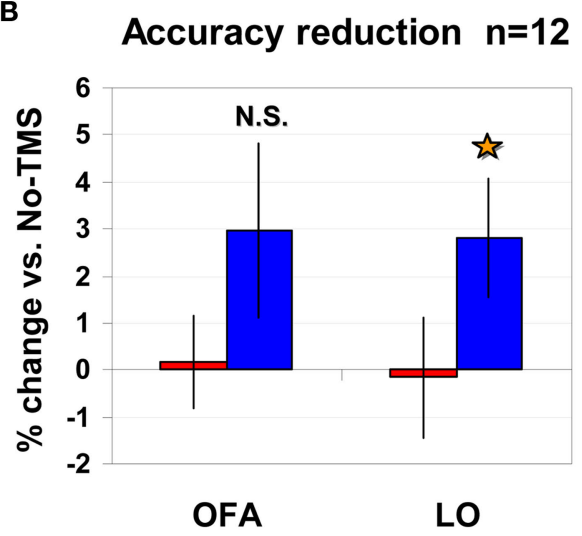

is $p<0.05$

N.S. $p>0.13$

FIGURE 4 | Accuracy levels according to trial type andTMS site. (A) No significant effects were found for either trial type or TMS site. (B) Planned comparisons of TMS conditions relative to No-TMS revealed a significant reduction in accuracy for LO-TMS, for repeated trials only. TMS over OFA did not induce significant changes in accuracy. Red-different identity trials, blue - repeated identity trials. Error bars, SEM.

image statistics differed widely across all images, in order to rule out any effects of low-level image similarities between exemplars of a single identity. We found a strong and consistent identity repetition modulation effect evident through shorter RTs for repetition of the same facial identity. TMS did not differentially affect repeated and different identities whether applied over OFA or LO while the repetition identity modulation effect remained robust. Our findings that the presentation of the first stimulus did not modulate the TMS effect on the second stimulus despite the matching identity suggests that the OFA does not contain neuronal representations that encode identity independently of low-level visual features. It is therefore more likely that the face-related activations observed in OFA and LO are related to processing of facial image features or generic aspects of faces. Future studies examining stimulus repetition effects of famous faces might reveal in more detail the role that OFA and LO play in facial representations.

\section{POSSIBLE MECHANISMS TO ACCOUNT FOR FACIAL IDENTITY REPETITION EFFECTS}

Repetition of a specific stimulus can cause behavioral facilitation due to previous exposure to that stimulus, and this phenomenon is known as priming (for review see Kristjansson and Campana, 2010). Here we showed that for the general concept of facial identity we found a similar phenomenon that was not bound to a specific physical stimulus but rather to the facial identity represented by that stimulus. We found a consistent identity repetition modulation effect, so that when the same identity was repeated (but with a different image), participants were faster to respond. It is important to emphasize that in order to respond faster to the same identity, participants had to process identity rather than simple image similarity, overcoming physical differences in low-level image properties between images that were as big as for different identities (see Figure 2). Hence, there are likely to be neuronal populations in the human brain that are tuned to identity and succeed in generalizing over numerous appearances of the same individual to allow participants to classify them as "same identity" out of the endless number of facial images they might encounter (Quiroga et al., 2005). We assume that when a face of a familiar person is observed, these identity-sensitive neuronal representations are activated, thus facilitating consequent processing of that same individual under different viewing conditions. Although images of the same individual might in general be more similar to each other than images of different individuals, in this experiment we ensured that in terms of image statistics the physical difference between two appearances of the same individual could be as large as for different individuals (Figures 2B,D). Thus the neural representation of identity was dissociated from the physical stimulus characteristics. Whether facial identity representation in the brain is view-based (Newell et al., 1999; Ullman and Bart, 2004; Fang et al., 2007), object-center based (McKone, 2008), or uses alternative dimensions to represent identity (Bart and Ullman, 2008), the human visual system evidently has sufficient computational power to achieve this (Levy et al., 2004). Although the FFA, OFA, and STS regions in visual cortex are candidate regions to participate in identity representation (Rotshtein et al., 2005; Winston et al., 2004), this does not imply that the process is achieved within the visual cortex and other regions are likely to participate in such a process (e.g., MTL, Quiroga et al., 2005) and frontal regions (Rotshtein et al., 2005; Kriegeskorte et al., 2007; Rolls, 2007). However, in this study we sought to investigate the regions early in the face network accessible to TMS to provide insight into their function.

\section{TMS-INDUCED EFFECTS FOR LO}

Occipital face area is considered face-selective (Halgren et al., 1999; Haxby et al., 1999; Gauthier et al., 2000b) while LO is primarily an object-selective area although also activated by faces (Avidan et al., 2002a,b; Gilaie-Dotan et al., 2010). We therefore expected OFA to be more sensitive to the application of TMS in a paradigm using face stimuli. Surprisingly, we found that the application of TMS over LO, but not over OFA, induced an RT facilitation relative to the No-TMS condition which was present for both repeated and different identity trials. Furthermore, a small but significant reduction in accuracy levels was found for repeated identities relative 
to the No-TMS condition only under LO-TMS. The lack of such modulations with OFA-TMS rules out the possibility that this effect was due to artifacts such as heightened alertness induced by the tapping sensation on subjects' scalps or coil clicks.

This TMS-induced RT facilitation may be due to the stimulation of shape-related representations in LO demonstrated by neuroimaging studies (Malach et al., 1995; Grill-Spector et al., 1998a; Lerner et al., 2001). These shape representations can vary in their holistic nature from shape features to a whole face (GrillSpector et al., 1998b; Lerner et al., 2001, 2008; Ullman et al., 2002), yet they do not seem to take part in face identity representation (i.e., generalize over identity). Such facilitations are often observed when TMS is applied prior to the onset of the test stimulus as was also the case in the present study (e.g., Topper et al., 1998; Grosbras and Paus, 2003; see Silvanto and Muggleton, 2008a for discussion). These facilitations are often explained in terms of TMS-induced non-specific increases in cortical excitability of the stimulated region that facilitates subsequent sensory processing. In this view, LO played a role in the processing of the face stimuli and this process was facilitated by the preceding TMS pulse train. Alternatively, the facilitatory effects of TMS could be due to TMS inducing a "virtual lesion" in LO, thus reducing inhibition on other regions which are in "competition" with LO (e.g., Walsh et al., 1998). Such "disinhibition" accounts tend to involve regions which are known to inhibit each other (such as the dorsal/ventral stream regions; cf. Walsh et al., 1998; and left and right parietal cortices; cf. Hilgetag et al., 2001). This account begs the question of which regions could be in competition with $\mathrm{LO}$. An explanation of these results in terms of disinhibition of OFA is ruled out by the fact that OFA-TMS had no effect in our task. It is also not clear why OFA should be in competition with other face-selective regions such as FFA.

However, the critical finding from the present study is that such RT facilitation was not mediated by facial identity, indicating that LO does not represent facial features independently of their low-level features. Further research will be needed to determine whether the effects we observed in LO are face-specific or rather shape- or stimulus-generic effects.

In our experiment, OFA and LO were defined on a per-participant basis and were clearly physically and functionally separable in each participant (see Table 2). Consistent with this successful anatomical segregation of TMS target sites, we also demonstrated significant differences in TMS effects over these sites compared to No-TMS with familiar face stimuli. The effect we observed here was specific to LO and not to OFA, demonstrating that we could observe differential TMS effects on occipital cortex despite the relative anatomical proximity of the target sites. In addition, a recent state-dependent TMS study shows that neural populations in OFA and LO can be successfully distinguished with respect to rotation invariance of two-dimensional shapes (Silvanto et al., in press). This confirms the functional segregation of the two areas as has been observed previously in TMS studies (Pitcher et al., 2009).

\section{THE FACE-SELECTIVITY OF OFA}

The face-selectivity of OFA has been demonstrated using several different approaches, e.g., fMRI studies (Gauthier et al., 2000b), TMS studies (Pitcher et al., 2009), and case studies (Rossion et al.,
2003; Steeves et al., 2006, 2009). However the role it plays in the face-processing network is not completely clear. While a few studies have shown different face-related effects in OFA and FFA (Rotshtein et al., 2005; Yovel and Kanwisher, 2005; Liu et al., 2009), other studies indicate similar sensitivities albeit to a noisier extent in OFA (Haxby et al., 1999; Gauthier et al., 2000b; Gilaie-Dotan et al., 2008). Hence, it is unclear what the face-selectivity of OFA conveys, and which aspects of facial image features OFA might be tuned to. Here we examined whether the face-selectivity might be related to the high-level concept of identity (see Rossion et al., 2003; Steeves et al., 2006, 2009) or to lower level facial or shape features. Our finding that there was no modulation of RT facilitation when TMS was applied over OFA by identity repetition, suggests that OFA faceselectivity is not directly related to facial identity representations.

Alternatively, OFA face-selectivity might be related to lower level face or shape features, or to the generic configural aspects of faces. In this case OFA tuning to lower level facial features could be stimulus specific showing no invariance to changes such as size, contrast, or even location. Alternatively, OFA tuning to such lower level facial features could show some invariance to the location of the feature in the image, or to contrast or size changes of these features. Our experimental design was not directly intended to distinguish between these two possibilities, and our facial stimuli were highly variable in all of these aspects (size, contrast, orientation, expression, and also location of facial features within the image). Thus, our failure to find any RT facilitation effect when TMS was applied over OFA may hint toward stimulus specific facial representations in OFA that are highly sensitive to image changes (Pitcher et al., 2007; Rotshtein et al., 2007; Liu et al., 2009). This could be tested in future work, for example by employing repeated images as baseline and imposing various feature manipulations, while comparing them to completely different facial images.

The OFA could also conceivably be affected by variability of the backgrounds in our stimuli. This was deliberately not controlled in order to maximize low-level differences across the stimuli (and hence emphasize the consistency of identity, the factor under study). In addition, the differences in absolute and relative anatomical location of OFA in different observers that has been consistently reported (cf. Gauthier et al., 2000a; Pitcher et al., 2009 with Grossman and Blake, 2002; Pitcher et al., 2008) could further indicate that the face-selectivity of OFA might be linked to basic facial image features. Variability in the location of OFA identified by functional localizers could arise from different activation peaks in functional localizer scans, each reflecting tuning of different neuronal populations to subtly different types of facial features. Since different studies employ different stimuli sets with different facial image characteristics, they could be activating closely adjacent peaks, each tuned to different types of facial features (Pitcher et al., 2007; Liu et al., 2009) and therefore contributing to variability in the functionally defined anatomical location of OFA.

One factor that is potentially relevant when considering neuronal representations in OFA is facial familiarity. Many of the studies examining face-selectivity define face-selectivity from responses to unfamiliar faces (e.g., Kanwisher et al., 1997; Haxby et al., 1999; Gauthier et al., 2000b). It is widely accepted that the neural representation of faces changes significantly as a function 
of familiarity (Gauthier et al., 2000b; Hasson et al., 2003; Rotshtein et al., 2005, 2007; Winston et al., 2004; Gilaie-Dotan and Malach, 2007; Viskontas et al., 2009), and this is also observed behaviorally (Hancock et al., 2000). This change in representation may partially account for differences between the present study and previous findings (Pitcher et al., 2009). In that earlier study discrimination of unfamiliar faces is affected when TMS is applied over OFA, but not over LO. However these previous reported effects are based on sensitivity levels, while no effects are found for RTs. Since that earlier study employed various facial morph levels, and the null RT result they report is based on an analysis that collapses all morph levels (including same and different), it is hard to compare the RT results we find when TMS is applied over LO with their null RT result with respect to LO. In addition, the studies also differ in other important ways with respect to the application of TMS. In the present study, we applied TMS during the delay period between first and second image presentations, while in the earlier study TMS was applied during the presentation of the second (target) image. Finally, the earlier study examined stimulus specific effects in unfamiliar faces that were confined to specific locations in the visual field and found an effect of OFA-TMS, while we examined identity representation effects for familiar faces regardless of image similarities and did not find a TMS effect over OFA. Thus, there are important differences between present and previous studies including stimulus familiarity, task differences (stimulus vs. identity discrimination), and TMS timing that might account for the differences in findings. Nevertheless, even despite these differences, when interpreting the results of the two studies with respect to the role of OFA in facial processing, they suggest that the face sensitivity of the OFA may be related to low-level features of faces and not to identity. Our results further indicate that for familiar faces this sensitivity to basic face features does not generalize to identity representation.

\section{REFERENCES}

Andrews, T. J., and Ewbank, M. P. (2004). Distinct representations for facial identity and changeable aspects of faces in the human temporal lobe. Neuroimage 23, 905-913.

Avidan, G., Harel, M., Hendler, T., BenBashat, D., Zohary, E., and Malach, R. (2002a). Contrast sensitivity in human visual areas and its relationship to object recognition. J. Neurophysiol. 87, 3102-3116.

Avidan, G., Hasson, U., Hendler, T., Zohary, U., and Malach, R. (2002b). Analysis of the neuronal selectivity underlying low fMRI signals. Curr. Biol. 12, 964-972.

Bart, E., and Ullman, S. (2008). Classbased feature matching across unrestricted transformations. IEEE Trans. Pattern Anal. Mach. Intell. 30, 1618-1631.

Campana, G., Cowey, A., and Walsh, V. (2002). Priming of motion direction and area V5/MT: a test of perceptual memory. Cereb. Cortex 12, 663-669.

Cattaneo, Z., Devlin, J. T., Salvini, F., Vecchi, T., and Silvanto, J. (2010). The causal role of category-specific neuronal representations in the left ventral premotor cortex $(\mathrm{PMv})$ in semantic processing. Neuroimage 49, 2728-2734.

Cattaneo, Z., Vecchi, T., Pascual-Leone, A., and Silvanto, J. (2009). Contrasting early visual cortical activation states causally involved in visual imagery and short-term memory. Eur. J. Neurosci. 30, 1393-1400.

Chen, C. C., Kao, K. L., and Tyler, C. W. (2007). Face configuration processing in the human brain: the role of symmetry. Cereb. Cortex 17, 1423-1432.

Cohen Kadosh, R., Muggleton, N., Silvanto, J., and Walsh, V. (2010). Double dissociation of format-dependent and number-specific neurons in human parietal cortex. Cereb. Cortex. doi: 10.1093/cercor/bHp273. [Epub ahead of print].

Dakin, S. C., and Watt, R. J. (2009). Biological "bar codes" in human faces. J. Vision 9, 1-10.

Fang, F., Murray, S. O., and He, S. (2007). Duration-dependent FMRI
Two case studies involving brain damage that encompasses OFA and display face recognition deficits suggest that OFA is involved in identity representation (Rossion et al., 2003; Steeves et al., 2006, 2009). However, lesions are rarely focal, and the functional borders of lesions are difficult to delineate. Therefore it is difficult to rule out that in each of these studies additional regions or connections that are associated with face processing have not been damaged. But even if a focal lesion confined to OFA would impair face recognition, this would still not be sufficient evidence for identity processing in OFA, but rather for its necessity in the process. Indeed, due to preliminary stages of face processing that are disrupted a face percept cannot be formed and consequently facial identity cannot be recognized.

Finally, it might be argued that our behavioral task was unable to reveal an effect of TMS on the facial identity repetition we observed due to a ceiling effect (as the accuracy was very high, $90 \%)$. This is unlikely for three reasons. Firstly, even though the accuracy levels were high, we found a small but significant sitespecific effect of TMS on accuracy levels, observed as reduction in accuracy in repeated identity trials only when TMS was applied over LO. Secondly, LO-TMS did induce a general effect on RTs, indicating the present task was sufficiently susceptible to TMS. And last, a previous study (Cattaneo et al., 2010) combining TMS with visual priming did find a differential TMS effect on the RTs of primed and non-primed trials, even though the overall accuracy was near $100 \%$. Therefore, high accuracy should not prevent the manifestation of state-dependent TMS effects on RTs.

\section{ACKNOWLEDGMENTS}

This work was supported by the Wellcome Trust (http://www. wellcome.ac.uk, http://www.fil.ion.ucl.ac.uk) and by a Marie-Curie fellowship to Sharon Gilaie-Dotan. adaptation and distributed viewercentered face representation in human visual cortex. Cereb. Cortex 17, 1402-1411.

Gauthier, I., Skudlarski, P., Gore, J. C., and Anderson, A. W. (2000a). Expertise for cars and birds recruits brain areas involved in face recognition. Nat. Neurosci. 3, 191-197.

Gauthier, I., Tarr, M. J., Moylan, J., Skudlarski, P., Gore, J. C., and Anderson,A.W. (2000b). The fusiform "face area" is part of a network that processes faces at the individual level. J. Cogn. Neurosci. 12, 495-504.

Gilaie-Dotan, S., Gelbard-Sagiv, H., and Malach, R. (2010). Perceptual shape sensitivity to upright and inverted faces is reflected in neuronal adaptation. Neuroimage 50, 383-395.

Gilaie-Dotan,S., and Malach, R. (2007).Subexemplar shape tuning in human facerelated areas. Cereb. Cortex 17, 325-338.

Gilaie-Dotan, S., Nir, Y., and Malach, R. (2008). Regionally-specific adaptation dynamics in human object areas. Neuroimage 39, 1926-1937.
Grill-Spector, K., Kushnir, T., Edelman, S., Itzchak, Y., and Malach, R. (1998a). Cue-invariant activation in object-related areas of the human occipital lobe. Neuron 21, 191-202.

Grill-Spector, K., Kushnir, T., Hendler, T., Edelman, S., Itzchak, Y., and Malach, R. (1998b). A sequence of objectprocessing stages revealed by $\mathrm{fMRI}$ in the human occipital lobe. Hum. Brain Mapp. 6, 316-328.

Grosbras, M. H., and Paus, T. (2003). Transcranial magnetic stimulation of the human frontal eye field facilitates visual awareness. Eur. J. Neurosci. 18, 3121-3126.

Grossman, E. D., and Blake, R. (2002). Brain areas active during visual perception of biological motion. Neuron 35, 1167-1175.

Halgren, E., Dale, A. M., Sereno, M. I., Tootell, R. B. H., Marinkovic, K., and Rosen, B. R. (1999). Location of human face-selective cortex with respect to retinotopic areas. Hum. Brain Mapp. 7, 29-37. 
Hancock, P. J., Bruce, V.V., and Burton, A. M. (2000). Recognition of unfamiliar faces. Trends Cogn. Sci. 4, 330-337.

Hasson, U., Harel, M., Levy, I., and Malach, R. (2003). Large-scale mirror-symmetry organization of human occipitotemporal object areas. Neuron 37, 1027-1041.

Haxby, J. V., Hoffman, E. A., and Gobbini, M. I. (2000). The distributed human neural system for face perception. Trends Cogn. Sci. 4, 223-233.

Haxby, J. V., Ungerleider, L. G., Clark, V. P., Schouten, J. L., Hoffman, E. A., and Martin, A. (1999). The effect of face inversion on activity in human neural systems for face and object perception. Neuron 22, 189-199.

Hilgetag, C. C., Theoret, H., and PascualLeone, A. (2001). Enhanced visual spatial attention ipsilateral to rTMSinduced 'virtual lesions' of human parietal cortex. Nat. Neurosci. 4, 953-957.

Johnston, R. A., and Barry, C. (2001). Best face forward: similarity effects in repetition priming of face recognition. $Q$. J. Exp. Psychol. A 54, 383-396.

Kanwisher, N., McDermott, J., and Chun, M. M. (1997). The fusiform face area: a module in human extrastriate cortex specialized for face perception. $J$. Neurosci. 17, 4302-4311.

Kriegeskorte, N., Formisano, E., Sorger, B., and Goebel, R. (2007). Individual faces elicit distinct response patterns in human anterior temporal cortex. Proc. Natl. Acad. Sci. U.S.A. 104, 20600-20605.

Kristjansson, A., and Campana, G. (2010). Where perception meets memory: a review of repetition priming in visual search tasks. Atten. Percept. Psychophys. 72, 5-18.

Lerner, Y., Epshtein, B., Ullman, S., and Malach, R. (2008). Class information predicts activation by object fragments in human object areas. J. Cogn. Neurosci. 20, 1189-1206.

Lerner, Y., Hendler, T., Ben-Bashat, D., Harel, M., and Malach, R. (2001). A hierarchical axis of object processing stages in the human visual cortex. Cereb. Cortex 11, 287-297.

Levy, I., Hasson, U., Avidan, G., Hendler, T., and Malach, R. (2001). Centerperiphery organization of human object areas. Nat. Neurosci. 4, 533-539.

Levy, I., Hasson, U., and Malach, R. (2004). One picture is worth at least a million neurons. Curr. Biol. 14, 996-1001.
Liu, J., Harris, A., and Kanwisher, N. (2009). Perception of face parts and face configurations: an fMRI study. J. Cogn. Neurosci. 22, 203-211.

Malach, R., Reppas, J. B., Benson, R. R., Kwong, K. K., Jiang, H., Kennedy, W. A., Ledden, P. J., Brady, T. J., Rosen, B. R., and Tootell, R. B. (1995). Objectrelated activity revealed by functional magnetic resonance imaging in human occipital cortex. Proc. Natl. Acad. Sci. U.S.A. 92, 8135-8139.

McCarthy, G., Puce, A., Gore, J. C., and Allison, T. (1997). Face specific processing in the human fusiform gyrus. J. Cogn. Neurosci. 9, 605-610.

McKone, E. (2008). Configural processing and face viewpoint. J. Exp. Psychol. Hum. Percept. Perform. 34, 310-327.

Newell, F. N., Chiroro, P., and Valentine, T. (1999). Recognizing unfamiliar faces: the effects of distinctiveness and view. Q. J. Exp. Psychol. A 52, 509-534.

Pitcher, D., Charles, L., Devlin, J. T., Walsh, V., and Duchaine, B. (2009). Triple dissociation of faces, bodies, and objects in extrastriate cortex. Curr. Biol. 19, 319-324.

Pitcher, D., Garrido, L., Walsh, V., and Duchaine, B. C. (2008). Transcranial magnetic stimulation disrupts the perception and embodiment of facial expressions. J. Neurosci. 28, 8929-8933.

Pitcher, D., Walsh, V., Yovel, G., and Duchaine, B. (2007). TMS evidence for the involvement of the right occipital face area in early face processing. Curr. Biol. 17, 1568-1573.

Pourtois, G., Schwartz, S., Seghier, M. L., Lazeyras, F., and Vuilleumier, P. (2005). Portraits or people? Distinct representations of face identity in the human visual cortex. J. Cogn. Neurosci. 17, 1043-1057.

Quiroga, R. Q., Reddy, L., Kreiman, G., Koch, C., and Fried, I. (2005). Invariant visual representation by single neurons in the human brain. Nature 435, 1102-1107.

Rhodes, G., Michie, P. T., Hughes, M. E., and Byatt, G. (2009). The fusiform face area and occipital face area show sensitivity to spatial relations in faces. Eur. J. Neurosci. 30, 721-733.

Rolls, E. T. (2007). The representation of information about faces in the temporal and frontal lobes. Neuropsychologia 45, 124-143.

Rossion, B., Caldara, R., Seghier, M., Schuller, A. M., Lazeyras, F., and Mayer, E. (2003). A network of occipito-tem- poral face-sensitive areas besides the right middle fusiform gyrus is necessary for normal face processing. Brain 126, 2381-2395.

Rotshtein, P., Geng, J. J., Driver, J., and Dolan, R. J. (2007). Role of features and second-order spatial relations in face discrimination, face recognition, and individual face skills: behavioral and functional magnetic resonance imaging data. J. Cogn. Neurosci. 19, 1435-1452.

Rotshtein, P., Henson, R. N., Treves, A., Driver, J., and Dolan, R. J. (2005). Morphing Marilyn into Maggie dissociates physical and identity face representations in the brain. Nat. Neurosci. 8, 107-113.

Silvanto, J., Lavie, N., and Walsh, V. (2005) Double dissociation of $\mathrm{V} 1$ and V5/MT activity in visual awareness. Cereb. Cortex 15, 1736-1741.

Silvanto, J., and Muggleton, N. G. (2008a) New light through old windows: moving beyond the "virtual lesion" approach to transcranial magnetic stimulation. Neuroimage 39, 549-552.

Silvanto, J., and Muggleton, N. G. (2008b). A novel approach for enhancing the functional specificity of TMS: revealing the properties of distinct neural populations within the stimulated region. Clin. Neurophysiol. 119 , 724-726.

Silvanto, J., Muggleton, N., and Walsh, V. (2008). State-dependency in brain stimulation studies of perception and cognition. Trends Cogn. Sci. 12, 447-454.

Silvanto, J., Schwarzkopf, D. S., GilaieDotan, S., and Rees, G. (in press). Differing causal roles for LO and OFA in invariant shape recognition. Eur. J. Neurosci.

Steeves, J., Dricot, L., Goltz, H. C., Sorger, B., Peters, J., Milner, A. D., Goodale, M. A., Goebel, R., and Rossion, B. (2009). Abnormal face identity coding in the middle fusiform gyrus of two brain-damaged prosopagnosic patients. Neuropsychologia 47, 2584-2592.

Steeves, J. K., Culham, J. C., Duchaine, B. C., Pratesi, C. C., Valyear, K. F., Schindler, I., Humphrey, G. K., Milner, A. D., and Goodale, M. A. (2006). The fusiform face area is not sufficient for face recognition: evidence from a patient with dense prosopagnosia and no occipital face area. Neuropsychologia 44, 594-609.
Topper, R., Mottaghy, F. M., Brugmann, M., Noth, J., and Huber, W. (1998). Facilitation of picture naming by focal transcranial magnetic stimulation of Wernicke's area. Exp. Brain Res. 121, 371-378.

Ullman, S., and Bart, E. (2004). Recognition invariance obtained by extended and invariant features. Neural Netw. 17, 833-848.

Ullman, S., Vidal-Naquet, M., and Sali, E. (2002).Visual features of intermediate complexity and their use in classification. Nat. Neurosci. 5, 682-687.

Viskontas, I. V., Quiroga, R. Q., and Fried, I. (2009). Human medial temporal lobe neurons respond preferentially to personally relevant images. Proc. Natl. Acad. Sci. U.S.A. 106, 21329-21334.

Walsh, V., Ellison, A., Battelli, L., and Cowey,A. (1998). Task-specific impairments and enhancements induced by magnetic stimulation of human visual area V5. Proc. R. Soc. Lond., B, Biol. Sci. 265, 537-543.

Winston, J. S., Henson, R. N., FineGoulden, M. R., and Dolan, R. J. (2004). fMRI-adaptation reveals dissociable neural representations of identity and expression in face perception. J. Neurophysiol. 92, 1830-1839.

Yovel, G., and Kanwisher, N. (2005). The neural basis of the behavioral face-inversion effect. Curr. Biol. 15, 2256-2262.

Conflict of Interest Statement: The authors declare that the research was conducted in the absence of any commercial or financial relationships that could be construed as a potential conflict of interest.

Received: 03 March 2010; paper pending published: 25 March 2010; accepted: 28 May 2010; published online: 28 June 2010.

Citation: Gilaie-Dotan S, Silvanto J, Schwarzkopf DS and Rees G (2010) Investigating representations of facial identity in human ventral visual cortex with transcranial magnetic stimulation. Front. Hum. Neurosci. 4:50. doi: 10.3389/ fnhum.2010.00050

Copyright (C) 2010 Gilaie-Dotan, Silvanto, Schwarzkopf and Rees. This is an openaccess article subject to an exclusive license agreement between the authors and the Frontiers Research Foundation, which permits unrestricted use, distribution, and reproduction in any medium, provided the original authors and source are credited. 